\title{
Presence, processing, and localization of mouse ADAM15 during sperm maturation and the role of its disintegrin domain during sperm-egg binding
}

\author{
Karina Pastén-Hidalgo ${ }^{1}$, Rosaura Hernández-Rivas², Ana Lilia Roa-Espitia ${ }^{1}$, Manuel Sánchez- \\ Gutiérrez $^{3}$, Francisco Martínez-Pérez ${ }^{4}$, Alma Olivia Monrroy ${ }^{1}$, Enrique O Hernández-González ${ }^{1}$ \\ and Adela Mújica ${ }^{1}$
}

${ }^{1}$ Departamento de Biología Celular and ${ }^{2}$ Departamento de Biomedicina Molecular, Centro de Investigación y de Estudios Avanzados del Instituto Politécnico Nacional No. 2508 (CINVESTAV-IPN), PC 07360, México, DF, México, ${ }^{3}$ Area Académica de Medicina, Instituto de Ciencias de la Salud, Universidad Autónoma del Estado de Hidalgo, PC 42090, Pachuca, Hgo. México and ${ }^{4}$ Posgrado en Ciencias Biológicas, Facultad de Ciencias, Universidad Nacional Autónoma de México (UNAM), PC 04510, México, DF, México

Correspondence should be addressed to A Mújica; Email: adelam@cell.cinvestav.mx

\begin{abstract}
Successful fertilization requires gametes to complete several stages, beginning with maturation and transport along the male and female reproductive tracts and ending with the interaction between the sperm and the egg. This last step involves sperm-egg adhesion and membrane fusion. ADAMs (disintegrin and metalloprotease domain proteins) are a family of membrane-anchored glycoproteins that are thought to play diverse roles in cell-cell adhesion through their interaction with integrins. This study analyzes the presence, location, processing, and possible role of ADAM15 in mouse sperm. The presence of ADAM15 in mouse spermatozoa was detected by Western blotting, which revealed that ADAM15 is post-translationally processed, during epididymal sperm maturation and the acrosome reaction. The $35 \mathrm{kDa}$ antigen present in the acrosome-reacted sperm is the last proteolytic product of the 110/75 kDa ADAM15 found in noncapacitated sperm. This $35 \mathrm{kDa}$ protein contains the disintegrin domain. By indirect immunofluorescence, ADAM15 was identified in the acrosomal region and along the flagellum of mouse spermatozoa. In acrosome-reacted sperm, ADAM15 was lost from the acrosomal region, but remained diffusely distributed throughout the head and flagellum. Furthermore, the ADAM15 disintegrin domain (RPPTDDCDLPEF) partially inhibited fusion and almost completely inhibited sperm-oolemma adhesion. In conclusion, our data indicate that ADAM15 is present in the testis and in spermatozoa from the caput, corpus, and cauda epididymis, as well as in non-capacitated and acrosome-reacted gametes. Results also indicate that ADAM15 is processed during epididymal maturation and acrosome reaction and that it may play a role during sperm-egg binding.

Reproduction (2008) 136 41-51
\end{abstract}

\section{Introduction}

Mammalian fertilization is a complex process, which is accomplished by several sequential steps, involving the recognition and binding of the sperm to the zona pellucida and the binding and fusion of the sperm-egg plasma membranes. Prior to the sperm-egg interaction, spermatozoa must undergo a series of modifications while traveling along the epididymis, followed by their capacitation and the acrosome reaction. The last two steps take place within the female reproductive tract (Yanagimachi 1994).

Regarding the mechanism and molecular components involved in the process of fertilization, there is a considerable amount of information. ADAMs (disintegrin and metalloprotease domain proteins) are among the candidates that may be the binding entities at the egg membrane surface (Almeida et al. 1995, Evans et al. 1997, Bigler et al. 2000, Evans 2001, Takahashi et al. 2001). ADAMs are transmembrane glycoproteins with a characteristic domain organization composed of a signal sequence, pro-, metalloprotease, and disintegrin domains, a cysteine-rich region, a transmembrane domain, and a cytoplasmic tail. This protein family is implicated in a number of cellular processes, which include fertilization, neurogenesis, and myogenesis, in the shedding of membrane-bound receptors and also in the activation of cytokines. Expression of ADAMs can vary considerably in mammals; many of them such as ADAMs 2, 7, 18, 20, 21, 29, and 30 are expressed 
exclusively in the testis. Other members (ADAMs 8, 9, $10,11,12,15,17,19,22,23,28$, and 33) manifest a broader distribution (reviewed by Seals \& Courtneidge 2003; for expression, refer to the table of ADAMs at http://www.people.virginia.edu/ jw7g/).

Earlier evidence suggested that ADAMs 1, 2, and 3, also referred to as fertilin $\alpha$, fertilin $\beta$ and cyritestin respectively, participate in fertilization (Cho et al. 1998, 2000, Shamsadin et al. 1999, Zhu et al. 2000, Nishimura et al. 2001). Fertilin, which is a heterodimeric protein complex composed of $\alpha$ and $\beta$ subunits present on the sperm surface, has been thought to mediate adhesion and fusion between the sperm and the egg plasma membranes (Blobel et al. 1992, Cho et al. 1998, Evans et al. 1998). In mice, two different isoforms of ADAM1, ADAM1a and ADAM1b, are synthesized in the testes, while only ADAM $1 b$ has been found on the plasma membrane of epididymal sperm (Kim et al. 2003). Recently, it has been shown that mutant male mice lacking ADAM1b are fertile and that sperm lacking ADAM $1 b$ can still bind to and fuse with eggs, demonstrating that ADAM1b and ADAM2 are not necessary for sperm-egg fusion, as was formerly proposed (Kim et al. 2006).

Fertilin is composed of two subunits, fertilin $\alpha$ and $\beta$ (Primakoff et al. 1987, Blobel et al. 1990, 1992, Wolfsberg et al. 1993). Both subunits consist of large precursors that are proteolytically processed during various physiological stages of the sperm, eliminating the fertilin pro- and metalloprotease domains. Fertilin $\alpha$ is processed within the testis (Lum \& Blobel 1997), whereas fertilin $\beta$ processing occurs during the transit of spermatozoa along the epididymis (Blobel et al. 1990, Hunnicutt et al. 1997). In mature spermatozoa, the fertilin heterodimer expresses the disintegrin domain at its $\mathrm{N}$-terminus in the case of both fertilin subunits.

There is evidence that ADAM disintegrin domains, behaving as integrin ligands, may be important for sperm-egg interaction (Bigler et al. 2000, Zhu et al. 2000, Takahashi et al. 2001). ADAM15 may be one of these ADAMs, although their identities have not been fully recognized. ADAM15 has been implicated in cell-cell and cell-matrix interactions and in the proteolysis of molecules on the cell surface or the extracellular matrix (Nath et al. 1999, Eto et al. 2000, Fourie et al. 2003, Seals \& Courtneidge 2003). The function of ADAM15 in cell-cell adhesion has been attributed to the disintegrin domain as integrin ligand (Seals \& Courtneidge 2003), while functions involving ectodomain processing have been attributed to its metalloprotease domain (Fourie et al. 2003). Besides this, Eto et al. (2002) demonstrated that the disintegrin domains from ADAM2 and ADAM15 inhibit the binding of sperm to mouse egg in a dose-dependent manner, whereas that from ADAM17 does not affect the sperm-egg binding.
Although this result may indicate a possible role played by the ADAM15 disintegrin domain in spermegg binding, the presence of ADAM15 in sperm has not yet been described.

The current work reports the presence of ADAM15 in mouse spermatozoa and describes how ADAM15 is processed during epididymal maturation and AR as well as the possible participation of ADAM15 in sperm-egg interaction.

\section{Results}

\section{ADAM15 is present in testicular cells}

Sperm ADAM proteins, with a potential role in fertilization, are first expressed as large precursors in the testis and are subsequently processed post-translationally by the proteolytic removal of the $\mathrm{N}$-terminal portion of the precursor, which includes the pro- and the metalloprotease domains, leading to membrane-bound molecules consisting of the disintegrin, cysteine-rich, and epidermal growth factor-like extracellular domains (Blobel 2000). RT-PCR assays were performed in order to investigate whether ADAM15 is expressed in mouse testicular cells. For this purpose, the conserved sequences between mouse Adam 15 and rat Adam15 were used to design primers and amplify this gene. A 1800 bp RT-PCR amplification product was obtained and then cloned and sequenced (GenBank accession number EF506571). Simultaneously and acting as a positive control, the Adam 15 gene from mouse heart was amplified and a product of similar size was obtained (Fig. 1B). The nucleotide sequence from mouse testis confirmed that the 1800 bp products encoded a protein that shows $100 \%$ identity with lung Adam 15 (NP_033744). These results led us to conclude that the identified testis mouse sequence corresponds to a bona fide member of the ADAM family of metalloproteinases, because this protein contains the multi-functional domains consisting of metalloprotease, disintegrin, and cysteine-rich domains. Western blot assays were performed in order to demonstrate the presence of complete ADAM15 protein. Two different antibodies were used for this purpose: one of these recognized the pro-domain $\left(\mathrm{NH}_{2}\right)$ and the other identified the carboxyl domain of ADAM15 (COOH). The antibody against the $\mathrm{N}$-terminal of ADAM15 showed that Jurkat (positive control) and testicular cells expressed the precursor protein of $110 \mathrm{kDa}$ (Fig. 1C), whereas the antibody against the C-terminal recognized the precursor protein $(110 \mathrm{kDa})$ as well as the ADAM15 processing form, consisting of about 75-85 kDa (Fig. 1C). Taken together, these results demonstrate that the complete ADAM15 protein is present in mouse testis and that it is processed in the testis. 
A

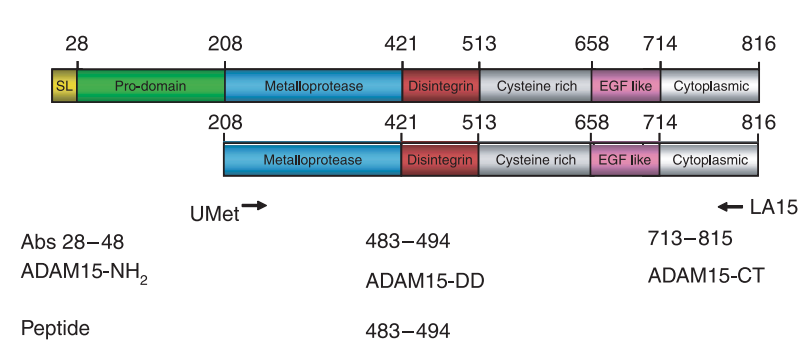

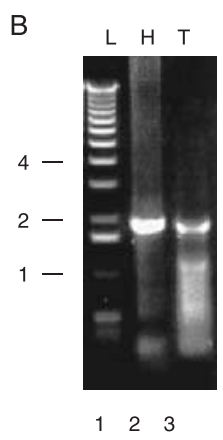

C

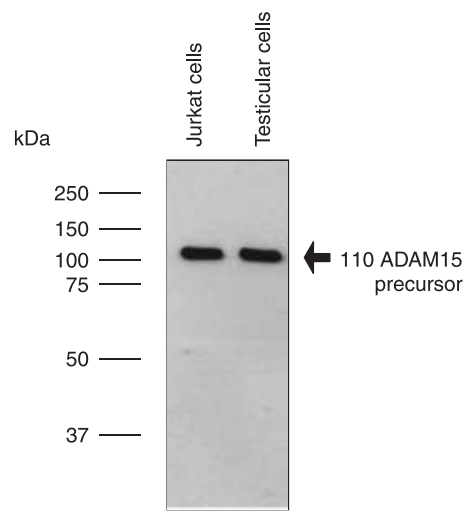

$\mathrm{NH}_{2}$

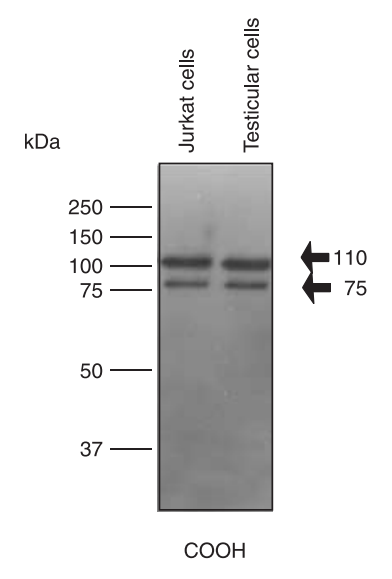

Figure 1 Cloning and expression of Adam15 cDNA from mouse testis. (A) Graphical representation of Adam 15 cDNA. The numbers correspond to the first amino acid that limits each domain. The named primers used in this work are indicated. The lines indicate the epitope recognized by each antibody (Abs) and the mapping region for the peptide used in this work. (B) RT-PCR products with UMet-LA15 from heart $(\mathrm{H})$ and testis $(\mathrm{T})$ were separated by electrophoresis in gel and visualized stained with ethidium bromide. The marker employed was $1 \mathrm{~Kb}$ DNA Ladder (L) (Invitrogen). Jurkat and testicular cell extracts are represented in (C). Cell proteins were dissolved in 1\% NP40 and subjected to SDS-PAGE, transferred to membranes, and blotted with anti-ADAM15-NT $\left(\mathrm{NH}_{2}\right)$ or with anti-ADAM15-CT $(\mathrm{COOH})$ antibodies. These antibodies recognize the pro-domain and cytoplasmic region of ADAM15 respectively. The molecular weight markers are indicated on the left.

\section{Sperm ADAM15 is processed during the gamete passage through the epididymis}

To determine whether mouse ADAM15 is processed during epididymal sperm maturation, protein extracts from the caput, corpus, and cauda epididymal mouse spermatozoa were analyzed by Western blot. An antibody against the carboxyl domain was used in order to identify the precursor $(110 \mathrm{kDa})$ and the processed form of ADAM15, because its processing occurs in the amino domain between the pro- and the metalloprotease domains (Herren et al. 1997, 2001, Poghosyan et al. 2002). ADAM15 precursor (about $110 \mathrm{kDa}$ ) was detected in spermatozoa proteins from the caput, corpus, and cauda epididymis. However, the precursor was most abundant in the caput epididymal sperm and its concentration progressively and significantly decreased along with sperm epididymal maturation, whereas a band of $75 \mathrm{kDa}$ was observed to progressively and significantly increase in concentration in caput, corpus, and cauda epididymis spermatozoa samples (Fig. 2A and C). A similar type of ADAM15 to that containing the metalloprotease domain was detected in HUVEC cells (Herren et al. 1997). These results indicate that ADAM15 is present as a large precursor and that it was proteolytically processed during sperm epididymal transit.
Moreover, in order to corroborate that ADAM15 is processed post-translationally by the proteolytic removal of the N-terminal during mouse sperm maturation, protein extracts from the epididymal sperm were analyzed by Western blot, using antibody against the ADAM15 $\left(\mathrm{NH}_{2}\right)$ pro-domain. This antibody recognized only a band of $110 \mathrm{kDa}$ in extracts from mouse epididymal sperm. Again, its concentration decreased during epididymal maturation of the mouse sperm (Fig. 2B and D). These results indicate that the ADAM15 pro-domain was processed during mouse sperm maturation. Western blot assays were performed to determine whether ADAM15 is processed during capacitation. The anti-ADAM15-COOH antibody detected a similar pattern observed in spermatozoa from the cauda epididymis (data not shown).

\section{Localization of ADAM15 in mouse spermatozoa}

In order to localize ADAM15 within mouse sperm, indirect immunofluorescence (IIF) assays with antiADAM15-CT antibody were performed in fixed and permeabilized spermatozoa during three physiological steps: the first sample was formed by non-capacitated sperm that are morphologically differentiated, but unable to fertilize; the second consisted of capacitated 

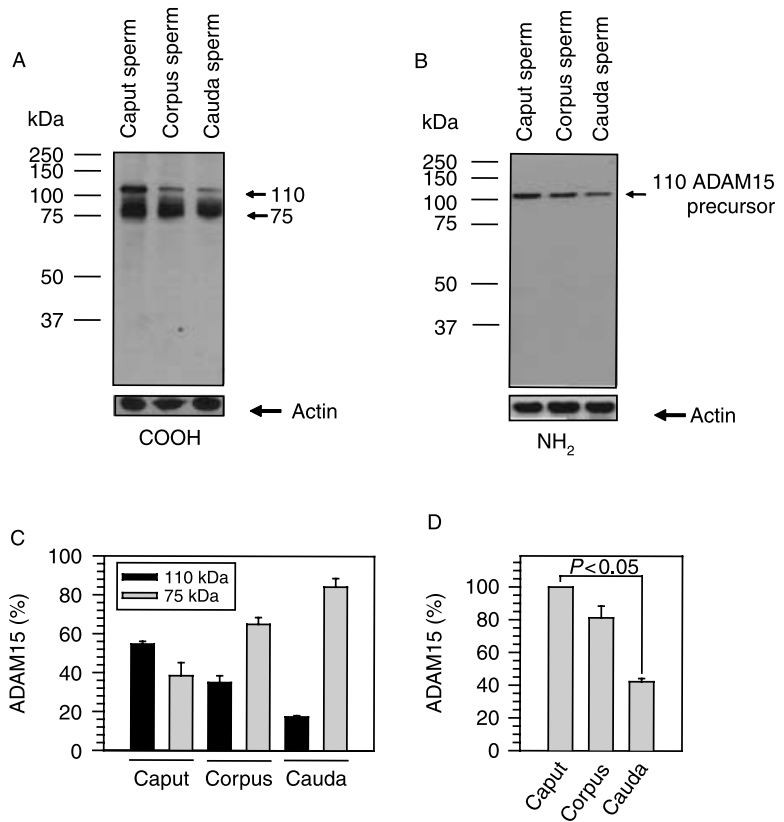

Figure 2 Mouse ADAM15 is processed during epididymal maturation. Cells at different developmental stages were lysed with 1\% NP-40. Proteins were boiled in Laemmli's buffer, subjected to SDS-PAGE, transferred to nitrocellulose membranes, and blotted with (A) antiADAM15-CT $(\mathrm{COOH})$ or (B) anti-ADAM15-NT $\left(\mathrm{NH}_{2}\right)$ antibodies. Protein $(60 \mu \mathrm{g})$ of the different epididymal sperm extracts (caput, corpus, and cauda) were applied per lane. Actin was used as a control to ensure the loading of the same protein amount in each lane. (C) Semi-quantitative analysis was carried out for the two ADAM15 forms ( 110 and $75 \mathrm{kDa}$ ) detected by the anti-ADAM15-CT antibody in caput, corpus, and cauda epididymal sperm. The mean of three measurements corrected for actin was calculated and expressed as a percentage of the ADAM15, where $100 \%$ is the sum of the two forms. (D) Semi-quantitative analysis of the ADAM15 recognized (110 kDa form) by the anti-ADAM15-NT antibody in caput, corpus, and cauda epididymal sperm. The mean of three measurements corrected for actin was calculated and expressed as a percentage of ADAM15 in caput sperm (100\%). (C and D) Values are means \pm S.E.M.; $n=3$.

sperm that are capable of binding to the ZP; and the third was formed by acrosome-reacted (AR) sperm that are able to pass through $\mathrm{ZP}$, and bind and fuse with the egg plasma membrane (Yanagimachi 1994). ADAM15 was located in the acrosome cap and flagellum of noncapacitated and capacitated sperm, still with acrosome (Fig. 3A and B respectively). When acrosomal exocytosis was induced with the calcium ionophore A23187, the pattern of fluorescence of the acrosome was lost, but the entire head and flagellum remained labeled diffusely (Fig. 3C). As expected, no signal was detected when pre-immune serum was used (Fig. 3D). The location of ADAM15 in the head of acrosome-reacted sperm suggests that ADAM15 may be involved in the binding of sperm to the egg plasma membrane. IIF assays were performed using antibody against the $\mathrm{N}$-terminal of the ADAM15, which recognized the prodomain: a similar pattern was detected in the samples from non-capacitated and capacitated sperm with acrosome reaction (Fig. $3 \mathrm{E}$ and $\mathrm{F}$ ), but only faint fluorescence. However, in acrosome-reacted sperm, the ADAM15 precursor was not detected (Fig. 3G). These results indicate that ADAM15 was present in mouse sperm and may undergo changes during the acrosome reaction.

\section{ADAM15 is further processed during $A R$}

In order to determine whether sperm ADAM15 is further modified during the acrosome reaction, immunoblot analysis were carried out on extracts from acrosomereacted mouse spermatozoa. The results indicated a change in molecular weight and size in mouse sperm 110 and $75 \mathrm{kDa}$ proteins yielding a $35 \mathrm{kDa}$ product during AR (Fig. 4A and B). These results demonstrate that in mouse spermatozoa ADAM15 was processed in the epididymis and then further processed during the acrosomal reaction. In order to determine whether the complete processing of ADAM15 produced membrane-bound molecules with a disintegrin extracellular domain, an antibody that recognized the active site of the disintegrin domain (DD) of ADAM15 was generated (this antibody was called ADAM15-DD). ADAM15-DD was used to analyze protein extracts from noncapacitated and acrosome-reacted sperm by Western blot. As shown in Fig. 4A and B, the ADAM15-DD antibody recognized the same bands as the ADAM15CT antibody in extracts from non-capacitated and acrosome-reacted mouse sperm. To corroborate and extend this result, IIF analysis in vivo (non-fixed and non-permeabilized cells) was performed. As shown in Fig. 4C, an immunopositive reaction was obtained in the acrosome cap and flagellum of noncapacitated cells, whereas in acrosome-reacted sperm the fluorescence pattern of the acrosome was lost although the head and flagellum remained labeled. The staining of spermatozoa with the ADAM15-DD antibody was abolished when it was pre-incubated with the ADAM15 peptide used to produce this antibody (Fig. 4C). When antibody against the ADAM15 carboxyl region was used, its location was the same regardless of whether the samples were fixed and permeabilized (see Fig. 3). These results showed that a processed form of ADAM15 $(35 \mathrm{kDa})$ is present in acrosome-reacted mouse sperm and that it contains an extracellular disintegrin domain.

\section{The ADAM15 disintegrin domain inhibited sperm-egg binding}

To define whether mouse ADAM15 was involved in sperm-egg binding, the active-site region of the mouse ADAM15 protein was defined by sequence alignment, using the snake disintegrin binding loop (Fig. 5A). 

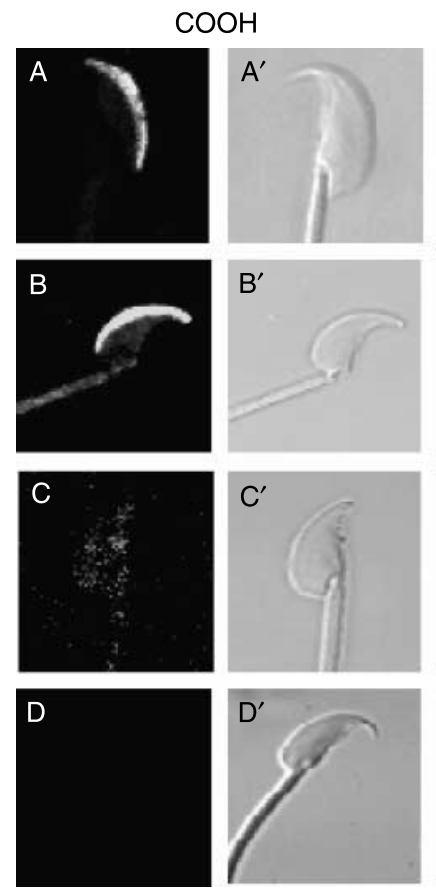

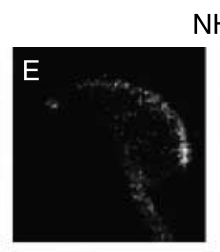

$\mathrm{NH}_{2}$
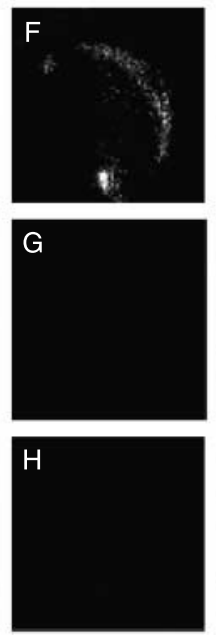

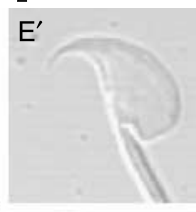

$F^{\prime}$
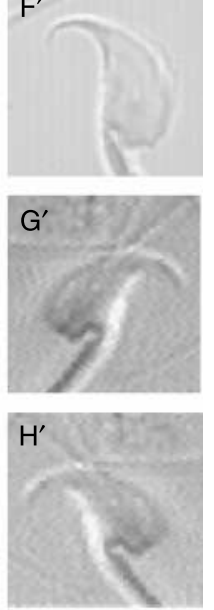

Non-capacitated

Acrosome reacted

Control

Capacitated

Following this, a peptide of 12 (483-RPPTDDCDLPEF-494) amino acid residues, corresponding to the predicted disintegrin loop of mouse ADAM15 was synthesized and used for competitive binding assays. This peptide inhibited the binding of sperm to zona-free mouse eggs in a dosedependent manner (Fig. 5B). Sperm-egg binding was inhibited $\sim 80 \%$ when $500 \mu \mathrm{M}$ dodecapeptide was used and $\sim 55 \%$ of sperm-egg fusion inhibition was observed at $300 \mu \mathrm{M}$ peptide (Fig. 5C). In order to evaluate the inhibition specificity obtained with this peptide, a control peptide was used: of the same composition but different sequence (DREPDLPCFDPT; McLaughlin et al. 2001) and no inhibition was observed (Fig. 5D). These results suggest that the disintegrin domain of ADAM15 may play a role in sperm-egg membrane adhesion.

\section{Discussion}

Here, we demonstrate that ADAM15 is present in mouse sperm, it is processed in the testis and during epididymal sperm maturation and it is further processed during the acrosomal reaction; also, ADAM15 participates in sperm-egg binding.

\section{Mouse testis ADAM15 contains the different domains present in ADAMs proteins}

The protein cloned in this work demonstrates that ADAM15 from mouse testis has multi-functional

domains consisting of a metalloprotease, a disintegrin, and a cysteine-rich region.

\section{Processing of ADAM15 during sperm epididymal maturation}

During the ADAMs protein maturation, proteolytic cleavage exposed different ADAMs domains, such as the metalloprotease or the disintegrin domain that potentially play a role in cell-surface proteolysis or cell adhesion (Seals \& Courtneidge 2003). ADAM15, a protein involved in cell-cell interaction (Nath et al. 1999, Eto et al. 2000), is known to lose the pro-domain while still inside the cell, probably when in the Golgi complex; ADAM15 is located on the plasma membrane, with the metalloprotease domain exposed (Lum et al. 1998). The present results analyze ADAM15 processing during mouse sperm maturation. Analysis of the proteins recognized by the antiADAM15 antibodies suggests that the pro-domain of ADAM15 is removed during processing in a manner similar to that of Testase 1 (Zhu et al. 2001). ADAM15 is partially processed in the mouse testis and cleaved after it has reached the sperm surface; this takes place during the sperm's transit through the epididymis. Consequently, metalloprotease and disintegrin domains of ADAM15 are present in cauda mouse sperm and non-capacitated sperm. ADAM15 is further processed during the acrosome reaction, possibly by acrosomal proteolytic enzymes, exposing the disintegrin domain.

When the pro- and the metalloprotease domains are absent in ADAM15, this molecule may play a role during 
A $\mathrm{kDa}$

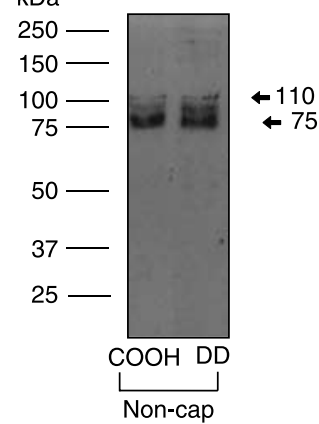

B $\mathrm{kDa}$

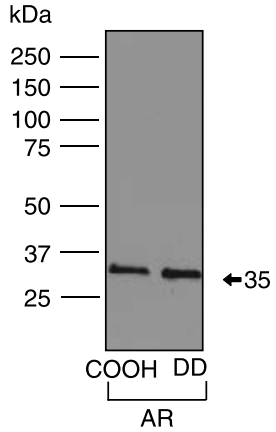

C

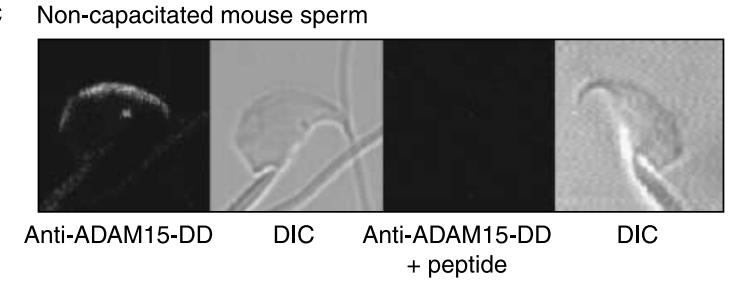

Acrosome-reacted mouse sperm

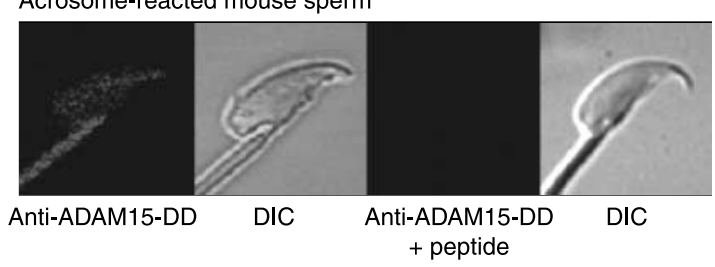

Figure 4 Mature ADAM15 from mouse sperm contains a disintegrin domain. Sperm proteins were dissolved in 1\% NP-40, subjected to SDS-PAGE, transferred to membranes, and blotted with anti-ADAM15-CT (COOH) or with ADAM15-DD (DD) antibodies. The molecular weight markers are indicated on the left. (A) Noncapacitated sperm and (B) acrosome-reacted sperm from mouse. (C) Images of indirect immunofluorescence of ADAM15 on live noncapacitated sperm $(n=81 / 90)$ and acrosome-reacted mouse sperm (85/90). The staining of mouse sperm with anti-ADAM15-DD antibody was abolished in the presence of the immunizing ADAM15 peptide. $\mathrm{DIC}$ represents the corresponding differential interface contrast of these cells.

sperm-egg binding through its disintegrin domain. However, what the function of ADAM15 is, prior to the acrosome reaction in mouse sperm, still remains unsolved. ADAM15 includes the metalloprotease domain that is activated by cleavage of the pro-domain (Lum et al. 1998). Primakoff has proposed that testase 1 has proteolytic activity on the sperm surface, which modifies other proteins during the later stages of epididymal maturation and/or fertilization (Zhu et al. 2001). Similar functions might be proposed for ADAM15.

Although the processing mechanism of ADAM15 is unknown, our results suggest that in mice, this processing requires alternative proteases. The ADAM 15 pro-domain may be processed by a pro-protein convertase, present in testis because ADAM15 contains a pro-protein convertase site, located between the proand metalloprotease domains. We suggest that the
ADAM15 metalloprotease domain in mouse sperm is removed in a way, similar to that of the metalloprotease domain of fertilin- $\alpha$. Blobel has proposed that the proprotein convertase PC4 may cleave the metalloprotease domain of fertilin- $\alpha$ (Lum \& Blobel 1997, Blobel 2000). The processing of ADAM15 during sperm maturation in the epididymis may be performed by proteases, secreted from the epididymis, although this still needs to be clarified because the proteases present in the epididymal fluid have not been extensively studied.

\section{ADAM15 may play a role in fertilization}

It has been postulated that at least three ADAMs participate in fertilization, ADAM1 (fertilin $\alpha$ ), ADAM2 (fertilin $\beta$ ), and ADAM3 (cyritestin). Studies employing synthetic peptides and anti-disintegrin loop antibodies appear to indicate that fertilin $\beta$ and cyritestin use their disintegrin domain to interact with the egg membrane (Evans 2001). This work upholds the premise that ADAM15 may also participate in the interaction between the sperm and the egg membrane because: (1) ADAM15 is present in mouse acrosome-reacted sperm at the point of fertilization; (2) following the acrosome reaction, ADAM 15 with its disintegrin domain exposed was located throughout the head, where the post-acrosomal region and equatorial segment are involved in the interaction of the sperm with the egg plasma membrane (Yanagimachi 1994); (3) the sequence determined for mouse ADAM15 indicates that the DCD motif is contained in its disintegrin domain; and (4) our assays for fertilization manifested the following results: the disintegrin peptide from mouse testis ADAM15 inhibited (80\%) sperm binding to zona-free mouse egg and sperm-egg fusion (55\%). This evidence points towards the possible participation of ADAM15 in the fertilization process. Similar results were presented for fertilin $\beta$ and cyritestin, using short fertilin $\beta$ or cyritestinbased peptide mimics, which have been found to cause very substantial inhibition of sperm-oocyte interactions. However, the gene knockout experiments in mice do not support this conclusion. For example, cyritestin-deficient sperm were still able to bind the oolemma (Shamsadin et al. 1999). McLaughlin et al. (2001) have proposed that these inconsistencies are due to the use of peptides that affected unintended targets. Furthermore, they present data indicating that a single XCD motif-containing peptide (where $X$ represents one of a limited, but as yet undefined, subset of amino acid residues) is able to inhibit mouse sperm-oolemma binding and fusion. These results indicate that the flanking sequences do not confer any sequence specificity in the in vitro assays. In our studies, which utilized a disintegrin ADAM15 peptide that contains the DCD motif, sperm-oolemma binding was affected, whereas a scrambled ADAM15 peptide that lacked the DCD inhibition failed to exhibit any effect. 
A
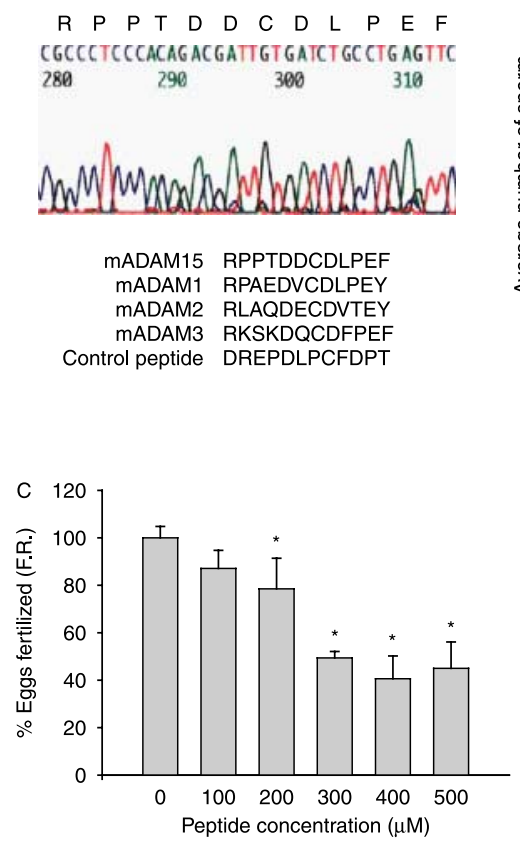

B
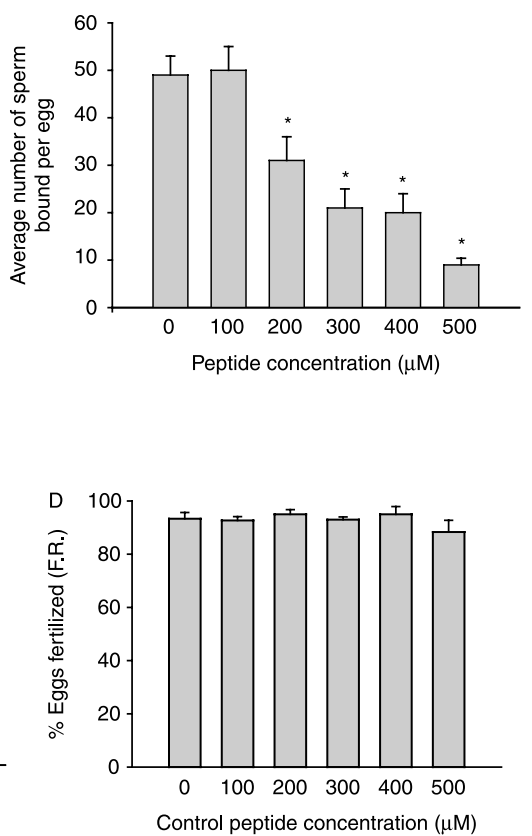

Figure 5 Effect of disintegrin peptide on sperm-egg binding and fusion. (A) Sequence comparison of known binding regions of small putative regions of disintegrin domains of mouse ADAM 1, 2, and 3 and ADAM15 peptides tested in this study. The ZP-free mouse eggs were incubated in the presence of the indicated concentrations $(\mu \mathrm{M})$ of peptide based on mouse ADAM15 (RPPTDDCDLPEF; see B and C), or control peptide (DREPDLPCFDPT; see D) for $30 \mathrm{~min}$. Then capacitated sperm were added and incubated for $30 \mathrm{~min}$ or $3-4 \mathrm{~h}$ at $37^{\circ} \mathrm{C}$, washed and analyzed for sperm binding (B) and fusion to the egg plasma membrane (C) and (D). Results present the average of four experiments per sample \pm S.E.M.; a total of 80 eggs were fertilized per sample. ${ }^{*} P<0.05$ versus control (without peptide).
These results implicate ADAM15 in binding to the oolemma, although still the in vivo role of ADAM15 remains to be defined because it is not known whether the peptide affects other targets. Regarding the possible role of ADAM15 in sperm-oolemma binding, we agree with the hypothesis forwarded by McLaughlin et al. (2001), which proposes that the concerted effects of several ADAMs result in maximum 'fertilizing ability' even if individual proteins are dispensable, implying some degree of functional redundancy.

The ADAMs disintegrin domains are homologous to snake venom disintegrins and represent potential integrin ligands. The disintegrin domain from human ADAM15 binds to the integrins $\alpha 9 \beta 1, \alpha v \beta 3$, and $\alpha v \beta 1$ (Zhang et al. 1998, Nath et al. 1999, Eto et al. 2000, 2002), but He et al. (2003) demonstrated that none of the integrins reported in mouse eggs or known as ADAM receptors are essential for sperm-egg binding and fusion. This finding raises the question whether other possible players are involved in the fertilization process.

ADAM15 is also present along the sperm flagellum, but its function is still unknown. As a rule, the entire sperm tail is incorporated into the egg (Yanagimachi 1994); therefore, the interaction that takes place between the plasma membranes from sperm tail and egg during fertilization may require the adhesion of molecules such as ADAM15.

In conclusion, this is the first time that ADAM15 has been observed in mammalian sperm, and our results, together with previously reported data (Eto et al. 2002), suggest the participation of ADAM15 in the fertilization process. Moreover, the role of ADAM15 in the human reproductive process should be considered of major relevance because the human spermatozoid does not express fertilin $\alpha$ or cyritestin (Jury et al. 1997, Frayne \& Hall 1998). The detection of ADAM15 opens new possibilities to improve our understanding of the mechanisms that regulate fertilization.

\section{Materials and Methods}

\section{Antibodies and reagents}

Goat polyclonal antibody anti-ADAM15-NT was purchased from Santa Cruz Biotechnology (Santa Cruz, CA, USA). Rabbit polyclonal antibody against the carboxyl terminal region of ADAM15 (anti-ADAM15-CT) was obtained from Oncogene Research Products (Boston, MA, USA). Dr J M Hernández (CINVESTAV, Mexico) kindly provided anti-actin MAB. HRP-conjugated anti-rabbit, HRP-conjugated antigoat, TRITC-conjugated anti-rabbit, and TRITC-conjugated anti-goat antibodies were purchased from Jackson ImmunoResearch (West Grove, PA, USA). DL-dithiothreitol, Trizma base, Tween-20, pepstatin, leupeptin, phenylmethylsulfonyl fluoride (PMSF), benzamidine, soybean trypsin inhibitor, and iodoacetamide were purchased from Sigma Chemical Co. Complete protease inhibitor cocktail was obtained from Roche. Nitrocellulose membrane was purchased from Bio-Rad. SDS was obtained from Calbiochem (San Diego, CA, USA). ECL detection kits were obtained from Amersham Pharmacia Biotechnology. Human chorionic gonadotropin (hCG), BSA fraction $\mathrm{V}$ (BSA), hyaluronidase were purchased from Sigma Chemical Co. Pregnant mare 
serum gonadotropin (PMSG) was obtained from INTERVET (Mexico).

\section{Adam15 cDNA from testis was cloned by RT-PCR}

Total RNA from NMRI mouse testis was isolated using the method described by Martinez-Perez et al. (2005). RT specific for Adam 15 mRNA was performed with $500 \mathrm{ng}$ primer LA15, $5 \mu \mathrm{g}$ total RNA from testis and ThermoScript Reverse Transcriptase (Invitrogen), following the manufacturer's instructions. Reaction conditions were $62{ }^{\circ} \mathrm{C}$ for $55 \mathrm{~min}$. The cDNA from heart was obtained, as previously described for external control RT from Adam15, and purified as reported by Martinez-Perez et al. (2005).

A 1800 bp DNA fragment of the Adam15 gene was obtained by means of RT-PCR, using the primers UMet-LA15 and LA15. These primers were designed in the function of the long Adam15 cDNA sequence from M. musculus (GenBank Accession number NM_009614, Fig. 1A). The PCR conditions consisted of 15 cycles of denaturing at $96^{\circ} \mathrm{C}$ for $20 \mathrm{~s}$, annealing at $55^{\circ} \mathrm{C}$ for $20 \mathrm{~s}$, and extending to $72{ }^{\circ} \mathrm{C}$ for $2.15 \mathrm{~min}$. Second touchdown was at $96^{\circ} \mathrm{C}$ for $15 \mathrm{~s}$, annealing at $60{ }^{\circ} \mathrm{C}$ for $15 \mathrm{~s}$, and extending to $72{ }^{\circ} \mathrm{C}$ for $2.15 \mathrm{~min}$, for 25 cycles. The PCR products were separated by electrophoresis in $1 \%$ agarose gels and visualized by staining the gel with ethidium bromide. The Adam15 RT-PCR products were excised and eluted from agarose, using silica gel particles, following the manufacturer's instructions (Quiagen).

To confirm the identity of the RT-PCR product as Adam15, a nested PCR was performed and each product was sequenced. The products were separated and visualized by electrophoresis in $2 \%$ agarose gel, as previously described. The RT-PCR products were purified using silica gel columns according to the manufacturer's instructions (Quiagen). Subsequently, they were subcloned in the vector PCR 4.0 with TOPO isomerase, using a TOPO TA cloning, following the manufacturer's instructions (Invitrogen). Ligation reaction was used to transform Escherichia coli DH $5 \alpha$ cells. The positive clones were purified and sequenced using a deoxy-sequencing kit (Biosystem Prysm Dye) and an ABI PRISM 310 Genetic Analyzer automatic sequencer (Applied Biosystems, Foster City, CA, USA). The nucleotide sequences were compared with other GenBank reported sequences, using the BLAST program. The conceptual translation was carried out with the program 'Traduccion multiple' from the infobiogen database (www.infobiogen.fr/).

\section{Peptides}

Peptides consisting of 12 amino acid residues that correspond to the predicted disintegrin loop of mouse ADAM15, as well as a scrambled sequence thereof, were prepared by (Invitrogen). The sequences were: ADAM15, RPPTDDCDLPEF; scrambled ADAM15, DREPDLPCFDPT.

\section{Antibody preparation}

A rabbit polyclonal antibody against the active site of the disintegrin domain of mouse ADAM15 (483RPPTDDCDLPEF494) was prepared in rabbits, using the peptide conjugated to BSA. A well-known immunization program was employed and the antibody was purified by means of affinity chromatography on a peptide-sepharose column (Drenckhahn et al. 1993). The antibody was called anti-ADAM15-DD.

\section{Preparations of testicular cells and epididymal sperm from mice}

Testicular cells were isolated from adult mice (12-week-old male NMR-1), as described by Phelps et al. (1990). Animals were anesthetized with chloroform and killed by cervical dislocation; the testis was quickly removed and stripped of its fat pad and epididymis. The tissue was chopped into small pieces using a razor blade and the fragments were vigorously pipetted using a wide bore pipette. The extract was filtered through a nylon mesh to remove connecting tissue and debris. After dissection, epididymal spermatozoa were removed from three regions of the epididymis: the caput, corpus, and cauda, and from vas deferens. Sperm were washed twice in PBS, by a process of resuspensioncentrifugation.

\section{In vitro capacitation and acrosome reaction in mice sperm}

Mouse sperm from the cauda epididymis and vas deferens were incubated at $37{ }^{\circ} \mathrm{C}$, under $5 \% \mathrm{CO}_{2}$ in air for $3 \mathrm{~h}$ in M16 medium, containing 3\% BSA for in vitro capacitation and acrosome reaction. In order to induce the acrosome reaction, spermatozoa were incubated for $15 \mathrm{~min}$ in M16 and then the medium was supplemented with $10 \mu \mathrm{M}$ of A23187 (Sigma Chemical Co.) for $15 \mathrm{~min}$. Successful induction of the acrosome reaction was verified by staining smears of formaldehyde-fixed spermatozoa, for 15-20 min with $0.2 \%$ Coomassie brilliant blue R250 (Larson \& Miller 1999). After washing with bi-distilled water, the stained spermatozoa were air dried and the preparation was mounted.

\section{Sperm immunofluorescence}

Non-capacitated sperm, capacitated gametes (with and without spontaneous acrosome reaction), and acrosome-reacted sperm, induced by a treatment with the calcium ionophore A23187, were fixed (v/v) with 3.0\% formaldehyde in PBS. Twenty minutes later, the spermatozoa were collected. Pelleted spermatozoa (at $600 \mathrm{~g}$ for $3 \mathrm{~min}$ ) were incubated in $50 \mathrm{mM}$ $\mathrm{NH}_{4} \mathrm{Cl}$ for $10 \mathrm{~min}$, then rinsed twice in PBS, and once in bi-distilled water and finally resuspended in bi-distilled water. This suspension was spread on microscope slides and air dried at room temperature. Subsequently, the cells were permeabilized with absolute acetone for $7 \mathrm{~min}$ at $-20{ }^{\circ} \mathrm{C}$ and washed in PBS. The presence of ADAM15 was revealed using antiADAM15-CT or with anti-ADAM-NT, both of which were diluted $(1: 100)$ in blocking solution, $1 \%$ BSA in PBS, and incubated for $1 \mathrm{~h}$ at $37^{\circ} \mathrm{C}$. Samples were washed in PBS, then incubated for $2 \mathrm{~h}$ at $37^{\circ} \mathrm{C}$ with the TRITC-conjugated antirabbit or TRITC-conjugated anti-goat antibodies, and diluted $(1: 100)$ in blocking solution. After being washed thrice in PBS, 
a drop of glycerol: PBS $(1: 1)$ was added to the samples that were placed on glass slides. These were covered with a coverslip and sealed with nail polish. Cells were viewed using a Zeiss photomicroscope equipped with phase-contrast and epifluorescent optics.

Live sperm were stained with anti-ADAM15-DD at a final dilution of 1:50, but they were neither permeabilized nor fixed. After a 2-h incubation at $37^{\circ} \mathrm{C}$ with the antibody, sperm samples were washed thrice with PBS. Goat anti-rabbit conjugated with rhodamine was added to the sperm in PBS, at a dilution of $1: 100$ and incubated for $1 \mathrm{~h}$ at $37^{\circ} \mathrm{C}$, together with antibody. Sperm were washed thoroughly with PBS beforehand and were later fixed with $1.5 \%$ formaldehyde for $10 \mathrm{~min}$. Microscopy slide smears were prepared and observed, as described previously.

\section{Western blot analysis}

Jurkat cells, testicular cells, epididymal sperm, and noncapacitated and acrosome-reacted sperm were collected by means of spin cell suspensions at $600 \mathrm{~g}$ for $3 \mathrm{~min}$. They were then washed twice with PBS, dissolved in lysis buffer (1\% NP40 in PBS; cell-extracts), and supplemented with the following set of protease inhibitors: $1 \mathrm{mM}$ PMSF, $2 \mu \mathrm{g} / \mathrm{ml}$ leupeptin, $2 \mu \mathrm{g} / \mathrm{ml}$ pepstatin, $50 \mu \mathrm{g} / \mathrm{ml}$ benzamidine, $1 \mu \mathrm{g} / \mathrm{ml}$ soybean trypsin inhibitor, $10 \mu \mathrm{g} / \mathrm{ml}$ iodoacetamide, and $300 \mu \mathrm{l}$ of a commercial mixture of protease inhibitors (a complete tablet dissolved in $\mathrm{H}_{2} \mathrm{O}$ ) for a $10 \mathrm{ml}$ sperm suspension. Samples were incubated on ice for $20 \mathrm{~min}$ and then centrifuged at $10000 \mathrm{~g}$ and $4{ }^{\circ} \mathrm{C}$ for $20 \mathrm{~min}$.

The supernatants were collected and their protein concentration was measured (Bradford 1976). Supernatant aliquots were boiled for $5 \mathrm{~min}$ in sample buffer (Laemmli 1970), containing $720 \mathrm{mM}$ of 2-mercaptoethanol for protein disulfide reduction and applied to SDS-PAGE gels. Following electrophoresis, the proteins were transferred to nitrocellulose membranes, which were then blocked using $5 \%$ fat-free dried milk in TBS-Tween $(10 \mathrm{mM}$ Tris- $\mathrm{HCl}$, $150 \mathrm{mM} \mathrm{NaCl}, 1 \%$ Tween $(\mathrm{pH}$ 7.5)). Membranes were incubated overnight at $4{ }^{\circ} \mathrm{C}$, along with anti-ADAM15-CT (1:500 dilutions), anti-ADAM15-NT (1:400 dilutions), antiADAM15-DD (1:100 dilutions), or anti-actin (1:100 dilutions). After five washes (7 min each) with TBS-Tween, the membranes were incubated with appropriate secondary antibodies, coupled to HRP (1:7500 dilutions). Immunereactive proteins were detected by chemiluminescence using an ECL kit.

\section{Superovulation and egg recovery}

Eggs were obtained from the oviducts of 6- to 8-week-old NMRI female mice. Superovulation was induced by s.c. injection of $15 \mathrm{IU}$ PMSG, followed by $15 \mathrm{IU}$ of hCG after a 48-h period. The animals were killed $15-17 \mathrm{~h}$ post-hCG. The uterine ovary-salpinge-horn complexes were dissected and eggs were removed from the ampulla of the oviduct and collected in M16 medium, supplemented with 3\% BSA. The ampulla of each oviduct was punctured, the cumulus mass extruded, and placed in $0.1 \%$ hyaluronidase in M16 medium for $7 \mathrm{~min}$ at $37^{\circ} \mathrm{C}$ in order to remove cumulus cells. Cumulus-free eggs were then washed four times in $\mathrm{M} 16$ medium, for 3 min each. The ZP was removed by incubating briefly in low pH buffer (Chen \& Sampson 1999). The ZP-free eggs were then immediately washed thrice with M16 medium and then the eggs were allowed to recover for $60 \mathrm{~min}$ in $\mathrm{M} 16$ medium at $37^{\circ} \mathrm{C}$ under $5 \% \mathrm{CO}_{2}$ /air. Only mature eggs with a detectable first polar body were used for fertilization.

\section{In vitro fertilization and assessment of sperm binding and fusion}

The ZP-free eggs were initially incubated with the peptide corresponding to the active site of the disintegrin domain of ADAM15 or with control peptide. Different peptide concentrations were assayed $(100-500 \mu \mathrm{M})$ in M16 medium with $3 \% \mathrm{BSA}, 30 \mathrm{~min}$ prior to co-incubation with capacitated sperm. None of the peptide concentrations tested altered sperm motility. Eggs and capacitated sperm (see above) were co-incubated in $200 \mu$ l drops (20 eggs per drop, with the addition of $10 \mu \mathrm{l}$ capacitated sperm suspension $\left(2 \times 10^{6}\right.$ cells $\left./ \mathrm{ml}\right)$. A 30 -min co-incubation period was employed for the determination of binding, but only after 3-4 h was it possible to clearly observe swollen sperm nuclei in the egg cytoplasm. Following the co-incubation of gametes, the eggs were washed thrice in M16 medium containing $3 \%$ BSA in order to remove any loosely attached sperm. Eggs were then fixed $(\mathrm{v} / \mathrm{v})$ with freshly prepared $3.0 \%$ formaldehyde in PBS for $1 \mathrm{~h}$. Eggs were washed twice in PBS and stained with Hoechst $33342(20 \mu \mathrm{g} / \mathrm{ml}$ PBS) for $30 \mathrm{~min}$, washed 3-4 times (15 min each time) in PBS, and mounted on a glass slide with $50 \%$ glycerol-PBS. Sperm-egg binding was observed in phasecontrast images. Fluorescent images of decondensed spermatozoa nuclei stained with Hoechst 33342 were recorded, in order to define whether the sperm had been incorporated into the egg cytoplasm.

\section{Statistical analyses}

Data were analyzed using a one-way ANOVA, followed by Tukey's honest significant differences and probability was set at $P<0.05$.

\section{Acknowledgements}

We thank Guadalupe Aguilar from Genetics and Molecular Biology Department for her technical assistance, Ivan J Galvan-Mendoza and Blanca Reyes for their confocal microscopy assistance and also Víctor Ramón Cortés for his technical assistance. We also thank Salvador Uribe for helpful comments on the manuscript. Isabel Pérez Montfort corrected the English version of the manuscript. Karina Pastén-Hidalgo was granted a doctoral fellowship by the Consejo Nacional de Ciencia y Tecnología (CONACyT No. 158648). This research was partially supported by a CONACYT grant (41725-Q) to A M. 


\section{Disclosure}

The authors declare that there is no conflict of interest that would prejudice the impartiality of this scientific work.

\section{References}

Almeida EA, Huovila AP, Sutherland AE, Stephens LE, Calarco PG, Shaw LM, Mercurio AM, Sonnenberg A, Primakoff P, Myles DG et al. 1995 Mouse egg integrin $\alpha 6 \beta 1$ functions as a sperm receptor. Cell 81 1095-1104.

Bigler D, Takahashi Y, Chen MS, Almeida EA, Osbourne L \& White JM 2000 Sequence-specific interaction between the disintegrin domain of mouse ADAM 2 (fertilin $\beta$ ) and murine eggs. Role of the $\alpha_{6}$ integrin subunit. Journal of Biological Chemistry 275 11576-11584.

Blobel CP 2000 Functional processing of fertilin: evidence for a critical role of proteolysis in sperm maturation and activation. Reviews of Reproduction 5 75-83.

Blobel CP, Myles DG, Primakoff P \& White JM 1990 Proteolytic processing of a protein involved in sperm-egg fusion correlates with acquisition of fertilization competence. Journal of Cell Biology 111 69-78.

Blobel CP, Wolfsberg TG, Turck CW, Myles DG, Primakoff P \& White JM 1992 A potential fusion peptide and an integrin ligand domain in a protein active in sperm-egg fusion. Nature 356 248-252.

Bradford MM 1976 A rapid and sensitive method for the quantitation of microgram quantities of protein utilizing the principle of protein-dye binding. Analytical Biochemistry 2 248-254.

Chen H \& Sampson NS 1999 Mediation of sperm-egg fusion: evidence that mouse egg $\alpha_{6} \beta_{1}$ integrin is the receptor for sperm fertilin $\beta$. Chemistry \& Biology 6 1-10.

Cho C, Bunch DO, Faure JE, Goulding EH, Eddy EM, Primakoff P \& Myles DG 1998 Fertilization defects in sperm from mice lacking fertilin B. Science 281 1857-1859.

Cho C, Ge H, Branciforte D, Primakoff P \& Myles DG 2000 Analysis of mouse fertilin in wild-type and fertilin $\beta^{-1-}$ sperm: evidence for C-terminal modification, $\alpha / \beta$ dimerization, and lack of essential role of fertilin $\alpha$ in sperm-egg fusion. Developmental Biology 222 289-295.

Drenckhahn D, Jöns T \& Schmitz F 1993 Production of polyclonal antibodies against proteins and peptides. In Methods in Cell Biology vol 37, pp 39-40. Ed. DJ Asai. California: Academic Press Inc.

Eto K, Puzon-McLaughlin W, Sheppard D, Sehara-Fujisawa A, Zhang XP \& Takada Y 2000 RGD-independent binding of integrin $\alpha_{9} \beta_{1}$ to the ADAM-12 and -15 disintegrin domains mediates cell-cell interaction. Journal of Biological Chemistry 275 34922-34930.

Eto K, Huet C, Tarui T, Kupriyanov S, Liu HZ, Puzon-McLaughlin W, Zhan XP, Sheppard D, Engvall E \& Takada Y 2002 Functional classification of ADAMs based on a conserved motif for binding to integrin $\alpha_{9} \beta_{1}$ implications for sperm-egg binding and other cell interactions. Journal of Biological Chemistry 277 17804-17810.

Evans JP 2001 Fertilin beta and other ADAMs as integrin ligands: insights into cell adhesion and fertilization. BioEssays 23 628-639.

Evans JP, Kopf GS \& Schultz RM 1997 Characterization of the binding of recombinant mouse sperm fertilin beta subunit to mouse eggs: evidence for adhesive activity via an egg $\beta 1$ integrin-mediated interaction. Developmental Biology 187 79-93.

Evans JP, Schultz RM \& Kopf GS 1998 Roles of the disintegrin domains of mouse fertilins $\alpha$ and $\beta$ in fertilization. Biology of Reproduction $\mathbf{5 9}$ 145-152.

Fourie AM, Coles F, Moreno V \& Karlsson L 2003 Catalytic activity of ADAM8, ADAM15, and MDC-L (ADAM28) on synthetic peptide substrates and in ectodomain cleavage of CD23. Journal of Biological Chemistry 278 30469-30477.

Frayne J \& Hall L 1998 The gene for the human tMDC I sperm surface protein is non-functional: implications for its proposed role in mammalian sperm-egg recognition. Biochemical Journal 334 171-176.

He ZY, Brakebusch C, Fässler R, Kreidberg JA, Primakoff P \& Myles DG 2003 None of the integrins known to be present on the mouse egg or to be ADAM receptors are essential for sperm-egg binding and fusion. Developmental Biology 254 226-237.
Herren B, Raines EW \& Ross R 1997 Expression of a disintegrin-like protein in cultured human vascular cells and in vivo. FASEB Journal $\mathbf{1 1}$ 173-180.

Herren B, Garton KJ, Coats S, Bowen-Pope DF, Ross R \& Raines EW 2001 ADAM15 overexpression in NIH3T3 cells enhances cell-cell interactions. Experimental Cell Research 271 152-160.

Hunnicutt GR, Koppel DE \& Myles DG 1997 Analysis of the process of localization of fertilin to the sperm posterior head plasma membrane domain during sperm maturation in the epididymis. Developmental Biology 191 146-159.

Jury JA, Frayne J \& Hall L 1997 The human fertilin $\alpha$ gene is non-functional: implications for its proposed role in fertilization. Biochemical Journal 321 577-581.

Kim E, Nishimura H \& Baba T 2003 Differential localization of ADAM1a and ADAM1b in the endoplasmic reticulum of testicular germ cells and on the surface of epididymal sperm. Biochemical and Biophysical Research Communications 304 313-319.

Kim E, Yamashita M, Nakanishi T, Park KE, Kimura M, Kashiwabara S \& Baba T 2006 Mouse sperm lacking ADAM1b/ADAM2 fertilin can fuse with the egg plasma membrane and effect fertilization. Journal of Biological Chemistry 281 5634-5639.

Laemmli UK 1970 Cleavage of structural proteins during the assembly of the head of bacteriophage T4. Nature 227 680-685.

Larson JL \& Miller DJ 1999 Simple histochemical stain for acrosomes on sperm from several species. Molecular Reproduction and Development 52 445-449.

Lum L \& Blobel CP 1997 Evidence for distinct serine protease activities with a potential role in processing the sperm protein fertilin. Developmental Biology 191 131-145.

Lum L, Reid MS \& Blobel CP 1998 Intracellular maturation of the mouse metalloprotease disintegrin MDC15. Journal of Biological Chemistry 273 26236-26247.

Martinez-Perez F, Zinker S, Aguilar G, Valdes J \& Arechiga H 2005 Circadian oscillations of RPCH gene expression in the eyestalk of the crayfish Cherax quadricarinatus. Peptides 12 2434-2444.

McLaughlin EA, Frayne J, Bloomerg G \& Hall L 2001 Do fertilin $\beta$ and cyritestin play a major role in mammalian sperm-oolemma interactions? A critical re-evaluation of the use of peptide mimics in identifying specific oocyte recognition proteins Molecular Human Reproduction 7 313-317.

Nath D, Slocombe PM, Stephens PE, Warn A, Hutchinson GR, Yamada KM, Docherty AJ \& Murphy G 1999 Interaction of metargidin (ADAM-15) with $\alpha_{v} \beta_{3}$ and $\alpha_{5} \beta_{1}$ integrins on different haemopoietic cells. Journal of Cell Science 112 579-587.

Nishimura H, Cho C, Branciforte DR, Myles DG \& Primakoff P 2001 Analysis of loss of adhesive function in sperm lacking cyritestin or fertilin B. Developmental Biology 233 204-213.

Phelps BM, Koppel DE, Primakoff P \& Myles DG 1990 Evidence that proteolysis of the surface is an initial step in the mechanism of formation of sperm cell surface domains. Journal of Cell Biology 111 1839-1847.

Poghosyan Z, Robbins SM, Houslay MD, Webster A, Murphy G \& Edwards DR 2002 Phosphorylation-dependent interactions between ADAM15 cytoplasmic domain and Src family protein-tyrosine kinases. Journal of Biological Chemistry 277 4999-5007.

Primakoff P, Hyatt H \& Tredick-Kline J 1987 Identification and purification of a sperm surface protein with a potential role in sperm-egg membrane fusion. Journal of Cell Biology 104 141-149.

Seals DF \& Courtneidge SA 2003 The ADAMs family of metalloproteases: multidomain proteins with multiple functions. Genes and Development 17 7-30.

Shamsadin R, Adham IM, Nayernia K, Heinlein UA, Oberwinkler H \& Engel W 1999 Male mice deficient for germ-cell cyritestin are infertile. Biology of Reproduction 61 1445-1451.

Takahashi Y, Bigler D, Ito Y \& White JM 2001 Sequence-specific interaction between the disintegrin domain of mouse ADAM 3 and murine eggs: role of $\beta 1$ integrin-associated proteins CD9, CD81, and CD98. Molecular Biology of the Cell 12 809-820.

Wolfsberg TG, Bazan JF, Blobel CP, Myles DG, Primakoff P \& White JM 1993 The precursor region of a protein active in sperm-egg fusion contains a metalloprotease and a disintegrin domain: structural, functional, and evolutionary implications. PNAS 90 10783-10787. 
Yanagimachi R 1994 Mammalian fertilization. In The Physiology of Reproduction, 5 edn, pp 189-317. Eds E Knobil \& JD Neill. New York: Raven Press.

Zhang XP, Kamata T, Yokoyama K, Puzon-McLaughlin W \& Takada Y 1998 Specific interaction of the recombinant disintegrin-like domain of MDC15 (Metargidin, ADAM-15) with integrin $\alpha \mathrm{v} \beta 3$. Journal of Biological Chemistry 273 7345-7350.

Zhu X, Bansal NP \& Evans JP 2000 Identification of key functional amino acids of the mouse fertilin $\beta$ (ADAM2) disintegrin loop for cell-cell adhesion during fertilization. Journal of Biological Chemistry 275 7677-7683.
Zhu GZ, Myles DG \& Primakoff P 2001 Testase 1 (ADAM 24) a plasma membrane-anchored sperm protease implicated in sperm function during epididymal maturation or fertilization. Journal of Cell Science 114 1787-1794.

Received 26 June 2007

First decision 7 September 2007

Revised manuscript received 11 March 2008

Accepted 26 March 2008 